\section{Crystal structure of methyl $N$-ferro- cenylcarbamate}

\section{Alan J. Rodríguez, ${ }^{\mathrm{a} *}$ J. Martin. E. Quirke ${ }^{\mathrm{a}}$ and Aida O. Diouf $^{b}$}

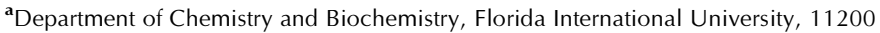
SW 8th Street, Miami, Florida 33199, USA, and ${ }^{\mathbf{b}}$ Department of Chemistry and Biochemistry, Albright College, 1621 North 13th Street, Reading, PA 19604, USA.

*Correspondence e-mail: arodr927@fiu.edu

Received 26 November 2014; accepted 2 January 2015
Triclinic, $P \overline{1}$

$a=10.1224(5) \AA$

$b=10.7849$ (5) $\AA$

$c=11.0445(5) \AA$

$\alpha=76.156$ (13) ${ }^{\circ}$

$\beta=73.960(13)^{\circ}$

$\gamma=89.059(14)^{\circ}$

$$
\begin{aligned}
& V=1123.52(9) \AA^{3} \\
& Z=4 \\
& \text { Mo } K \alpha \text { radiation } \\
& \mu=1.33 \mathrm{~mm}^{-1} \\
& T=296 \mathrm{~K} \\
& 0.38 \times 0.20 \times 0.15 \mathrm{~mm}
\end{aligned}
$$

\subsection{Data collection}

Bruker D8 Quest diffractometer Absorption correction: multi-scan (SADABS; Bruker, 2013)

$T_{\min }=0.75, T_{\max }=0.83$

24698 measured reflections 5586 independent reflections 4560 reflections with $I>2 \sigma(I)$

\subsection{Refinement}

$R\left[F^{2}>2 \sigma\left(F^{2}\right)\right]=0.030$

$w R\left(F^{2}\right)=0.071$

$S=0.99$

5586 reflections

299 parameters $R_{\text {int }}=0.022$
$\mathrm{H}$ atoms treated by a mixture of independent and constrained refinement

$\Delta \rho_{\max }=0.33{\mathrm{e} \AA^{-3}}^{-3}$

$\Delta \rho_{\min }=-0.26 \mathrm{e} \AA^{-3}$
Edited by T. J. Prior, University of Hull, England

The asymmetric unit of the title compound, $\left[\mathrm{Fe}\left(\mathrm{C}_{5} \mathrm{H}_{5}\right)\left(\mathrm{C}_{7} \mathrm{H}_{8} \mathrm{NO}_{2}\right)\right]$, contains two independent molecules consisting of a ferrocenyl moiety and a nitrogen-bound methyl carbamate. These units are almost perpendicular to each other, making dihedral angles of $87.74(9)$ and $87.32(8)^{\circ}$. In each independent molecule, the cyclopentadienyl rings deviate slightly from an eclipsed conformation and lie virtually parallel [dihedral angles $=1.42(15)$ and $0.49(13)^{\circ}$ ]. In the crystal, molecules are linked by $\mathrm{N}-\mathrm{H} \cdots \mathrm{O}$ hydrogen bonds into chains along the $a$-axis direction.

Keywords: crystal structure; ferrocene; carbamate; ferrocenoyl azide derivatives; $\mathrm{N}$-ferrocenoylmethylcarbamate.

CCDC reference: 1041904

\section{Related literature}

For the synthesis and fragmentation mechanism of the title compound, see: Van Berkel et al. (1998); Quirke et al. (2001). For related ferrocenyl derivatives, see: Barišić et al. (2011).

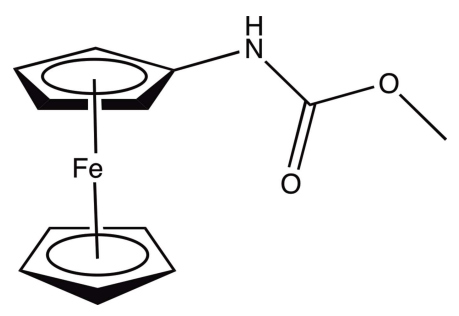

\section{Experimental}

2.1. Crystal data

$\left[\mathrm{Fe}\left(\mathrm{C}_{5} \mathrm{H}_{5}\right)\left(\mathrm{C}_{7} \mathrm{H}_{8} \mathrm{NO}_{2}\right)\right] \quad M_{r}=259.08$
Table 1

Hydrogen-bond geometry $\left(\AA{ }^{\circ}\right)$.

\begin{tabular}{lllll}
\hline$D-\mathrm{H} \cdots A$ & $D-\mathrm{H}$ & $\mathrm{H} \cdots A$ & $D \cdots A$ & $D-\mathrm{H} \cdots A$ \\
\hline $\mathrm{N} 1-\mathrm{H} 1 \cdots \mathrm{O} 3$ & \\
$\mathrm{~N} 2-\mathrm{H} 2 \cdots \mathrm{O} 1$ & $0.82(2)$ & $2.18(2)$ & $2.971(2)$ & $162.5(19)$ \\
\hline
\end{tabular}

Symmetry code: (i) $x+1, y, z$.

Data collection: APEX2 (Bruker, 2014); cell refinement: SAINT (Bruker, 2013); data reduction: $S A I N T$; program(s) used to solve structure: SHELXT-2014 (Sheldrick, 2015); program(s) used to refine structure: SHELXL2014/6 (Sheldrick, 2008); molecular graphics: Mercury (Macrae et al., 2006); software used to prepare material for publication: publCIF (Westrip, 2010).

\section{Acknowledgements}

The authors are grateful to R. G. Raptis for access to the X-ray diffractometer. AOD thanks the National Science Foundation - Research Experience for Undergraduates (NSF-REU, Award No. 1156886) for financial support.

Supporting information for this paper is available from the IUCr electronic archives (Reference: PJ2017).

\section{References}

Barišić, L., Roščić, M., Kovačević, M., Semenčić, M. M. C., Horvat, Š. \& Rapić, V. (2011). Carbohydr. Res. 346, 678-684.

Bruker (2013). SAINT and SADABS. Bruker AXS Inc., Madison, Wisconsin, USA.

Bruker (2014). APEX2. Bruker AXS Inc., Madison, Wisconsin, USA.

Macrae, C. F., Edgington, P. R., McCabe, P., Pidcock, E., Shields, G. P., Taylor, R., Towler, M. \& van de Streek, J. (2006). J. Appl. Cryst. 39, 453-457.

Quirke, J. M. E. \& Van Berkel, G. J. (2001). J. Mass Spectrom. 36, 179-187.

Sheldrick, G. M. (2008). Acta Cryst. A64, 112-122.

Sheldrick, G. M. (2015). Acta Cryst. C71, 3-8.

Van Berkel, G. J., Quirke, J. M. E., Tigani, R. A., Dilley, A. S. \& Covey, T. R. (1998). Anal. Chem. 70, 1544-1554.

Westrip, S. P. (2010). J. Appl. Cryst. 43, 920-925. 


\section{supporting information}

Acta Cryst. (2015). E71, m30 [doi:10.1107/S2056989015000043]

\section{Crystal structure of methyl $\mathrm{N}$-ferrocenylcarbamate}

\section{Alan J. Rodríguez, J. Martin. E. Quirke and Aida O. Diouf}



\section{Figure 1}

The molecular structure of the title compound with atoms drawn as ellipsoids at the $30 \%$ probability level. 


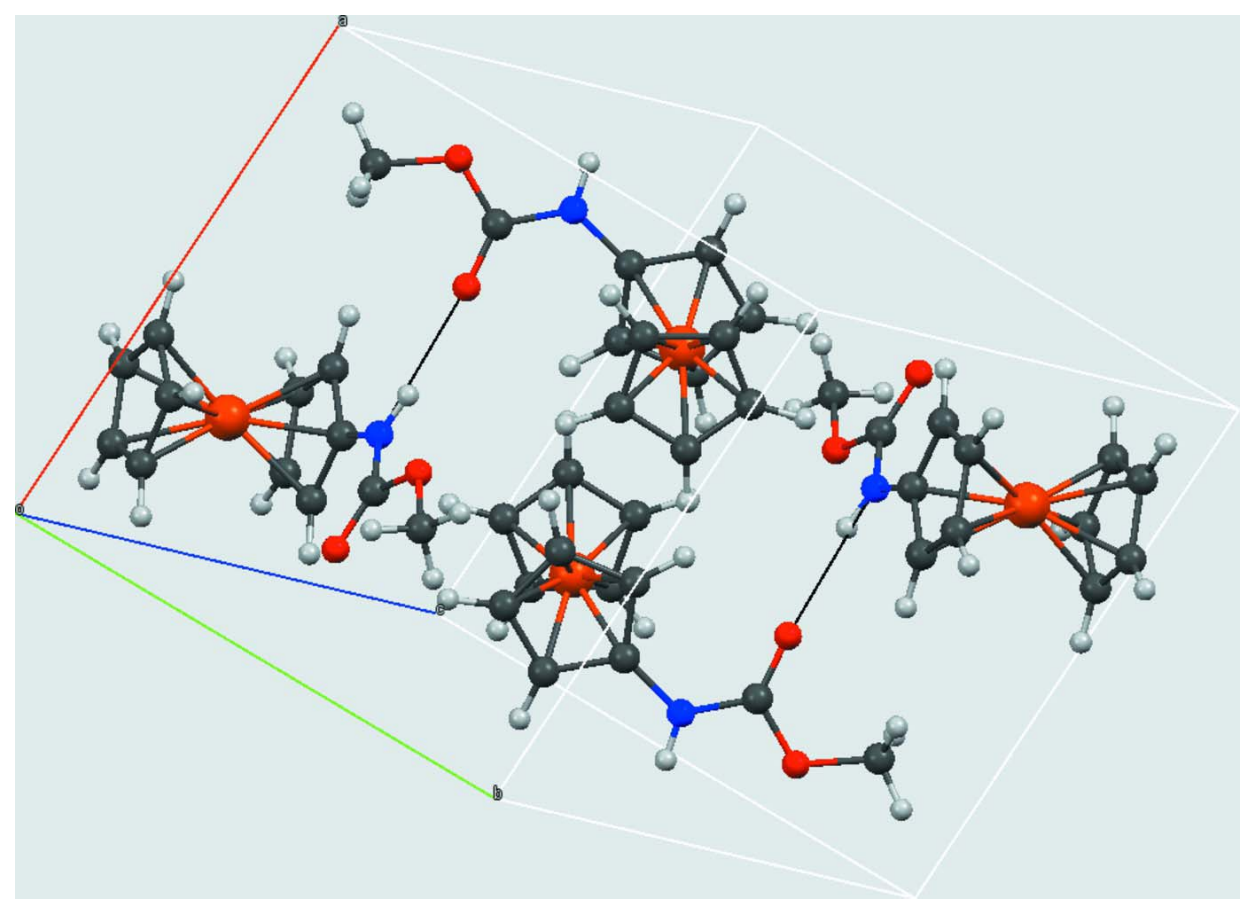

\section{Figure 2}

Packing diagram of the title compound showing intermolecular H-bonding interactions.

\section{Methyl $N$-ferrocenylcarbamate}

\section{Crystal data}

$\left[\mathrm{Fe}\left(\mathrm{C}_{5} \mathrm{H}_{5}\right)\left(\mathrm{C}_{7} \mathrm{H}_{8} \mathrm{NO}_{2}\right)\right]$

$M_{r}=259.08$

Triclinic, $P \overline{1}$

$a=10.1224(5) \AA$

$b=10.7849(5) \AA$

$c=11.0445(5) \AA$

$\alpha=76.156(13)^{\circ}$

$\beta=73.960(13)^{\circ}$

$\gamma=89.059(14)^{\circ}$

$V=1123.52(9) \AA^{3}$

\section{Data collection}

\section{Bruker D8 Quest}

diffractometer

Radiation source: fine-focus tube

Detector resolution: 10.4167 pixels $\mathrm{mm}^{-1}$

$\omega$ scans

Absorption correction: multi-scan

(SADABS; Bruker, 2013)

$T_{\min }=0.75, T_{\max }=0.83$

\section{Refinement}

Refinement on $F^{2}$

Least-squares matrix: full

$R\left[F^{2}>2 \sigma\left(F^{2}\right)\right]=0.030$
$Z=4$

$F(000)=536$

$D_{\mathrm{x}}=1.532 \mathrm{Mg} \mathrm{m}^{-3}$

Mo $K \alpha$ radiation, $\lambda=0.71073 \AA$

Cell parameters from 120 reflections

$\theta=2.9-22.8^{\circ}$

$\mu=1.33 \mathrm{~mm}^{-1}$

$T=296 \mathrm{~K}$

Needle, lusterous yellow

$0.38 \times 0.20 \times 0.15 \mathrm{~mm}$

24698 measured reflections

5586 independent reflections

4560 reflections with $I>2 \sigma(I)$

$R_{\text {int }}=0.022$

$\theta_{\text {max }}=28.3^{\circ}, \theta_{\text {min }}=2.9^{\circ}$

$h=-13 \rightarrow 13$

$k=-14 \rightarrow 14$

$l=-14 \rightarrow 14$

$w R\left(F^{2}\right)=0.071$

$S=0.99$

5586 reflections 
299 parameters

0 restraints

Hydrogen site location: mixed

$\mathrm{H}$ atoms treated by a mixture of independent

and constrained refinement

$$
\begin{aligned}
& w=1 /\left[\sigma^{2}\left(F_{\mathrm{o}}^{2}\right)+(0.0293 P)^{2}+0.5563 P\right] \\
& \text { where } P=\left(F_{\mathrm{o}}^{2}+2 F_{\mathrm{c}}^{2}\right) / 3 \\
& (\Delta / \sigma)_{\max }=0.001 \\
& \Delta \rho_{\max }=0.33 \mathrm{e} \AA^{-3} \\
& \Delta \rho_{\min }=-0.26 \mathrm{e} \AA^{-3}
\end{aligned}
$$

Special details

Geometry. All e.s.d.'s (except the e.s.d. in the dihedral angle between two 1.s. planes) are estimated using the full

\begin{tabular}{|c|c|c|c|c|}
\hline & $x$ & $y$ & $z$ & $U_{\text {iso }} * / U_{\text {eq }}$ \\
\hline $\mathrm{Fe} 1$ & $0.75463(2)$ & $0.60308(2)$ & $0.31717(2)$ & $0.03709(7)$ \\
\hline $\mathrm{Fe} 2$ & $0.25564(2)$ & $-0.00924(2)$ & $0.31751(2)$ & $0.03430(7)$ \\
\hline $\mathrm{C} 1$ & $0.82395(18)$ & $0.42097(17)$ & $0.34739(17)$ & $0.0389(4)$ \\
\hline $\mathrm{C} 2$ & $0.6871(2)$ & $0.42219(18)$ & $0.42686(18)$ & $0.0453(4)$ \\
\hline $\mathrm{H} 2 \mathrm{~A}$ & 0.6064 & 0.3734 & 0.4264 & $0.054 *$ \\
\hline $\mathrm{C} 3$ & $0.6888(2)$ & $0.5061(2)$ & 0.50739 (19) & $0.0546(5)$ \\
\hline H3 & 0.6091 & 0.5257 & 0.5726 & $0.065^{*}$ \\
\hline $\mathrm{C} 4$ & $0.8241(2)$ & $0.5576(2)$ & $0.4772(2)$ & 0.0560 \\
\hline $\mathrm{H} 4$ & 0.8548 & 0.6186 & 0.5181 & $0.067 *$ \\
\hline $\mathrm{C} 5$ & $0.9086(2)$ & $0.5057(2)$ & $0.3776(2)$ & $0.0485(5)$ \\
\hline H5 & 1.0077 & 0.5243 & 0.3376 & $0.058 *$ \\
\hline C6 & $0.6410(3)$ & $0.6599(2)$ & $0.1906(3)$ & $0.0737(8)$ \\
\hline H6 & 0.5717 & 0.6071 & 0.1761 & $0.088 *$ \\
\hline $\mathrm{C} 7$ & $0.6172(3)$ & $0.7354(3)$ & $0.2817(3)$ & $0.0780(8)$ \\
\hline $\mathrm{H} 7$ & 0.5283 & 0.7447 & 0.3419 & $0.094 *$ \\
\hline $\mathrm{C} 8$ & 0.7425 & $0.7959(2)$ & $0.2696(3)$ & $0.0750(8)$ \\
\hline $\mathrm{H} 8$ & 0.7574 & 0.8549 & 0.3202 & $0.090 *$ \\
\hline C9 & $0.8412(3)$ & $0.7591(2)$ & $0.1727(2)$ & $0.0691(7)$ \\
\hline H9 & 0.9390 & 0.7868 & 0.1439 & $0.083^{*}$ \\
\hline $\mathrm{C} 10$ & 0.7808 & $0.6751(2)$ & $0.1237(2)$ & $0.0684(7)$ \\
\hline H10 & 0.8276 & 0.6346 & 0.0541 & $0.082 *$ \\
\hline $\mathrm{C} 13$ & $0.32117(17)$ & $0.15345(17)$ & $0.35388(16)$ & $0.0372(4)$ \\
\hline $\mathrm{C} 14$ & $0.18305(18)$ & $0.11686(18)$ & $0.42921(17)$ & $0.0425(4)$ \\
\hline $\mathrm{H} 14$ & 0.1028 & 0.1685 & 0.4272 & $0.051^{*}$ \\
\hline C15 & $0.1830(2)$ & $-0.0073(2)$ & $0.50823(18)$ & $0.0505(5)$ \\
\hline $\mathrm{H} 15$ & 0.1021 & -0.0572 & 0.5706 & $0.061 *$ \\
\hline C16 & $0.3191(2)$ & $-0.0481(2)$ & 0.48139 (18) & $0.0502(5)$ \\
\hline H16 & 0.3490 & -0.1306 & 0.5222 & $0.060 *$ \\
\hline C17 & $0.40510(19)$ & $0.05143(18)$ & $0.38491(18)$ & $0.0438(4)$ \\
\hline $\mathrm{H} 17$ & 0.5047 & 0.0500 & 0.3472 & $0.053^{*}$ \\
\hline $\mathrm{C} 18$ & $0.2790(3)$ & $0.0257(2)$ & $0.12343(19)$ & $0.0569(5)$ \\
\hline H18 & 0.3187 & 0.1045 & 0.0583 & $0.068 *$ \\
\hline C19 & $0.1395(2)$ & -0.0010 & 0.1929 (2) & $0.0633(7)$ \\
\hline H19 & 0.0644 & 0.0561 & 0.1849 & $0.076 *$ \\
\hline
\end{tabular}
covariance matrix. The cell e.s.d.'s are taken into account individually in the estimation of e.s.d.'s in distances, angles and torsion angles; correlations between e.s.d.'s in cell parameters are only used when they are defined by crystal symmetry. An approximate (isotropic) treatment of cell e.s.d.'s is used for estimating e.s.d.'s involving l.s. planes.

Fractional atomic coordinates and isotropic or equivalent isotropic displacement parameters $\left(\AA^{2}\right)$ 


$\begin{array}{lllll}\mathrm{C} 20 & 0.1266(2) & -0.1246(3) & 0.2754(2) & 0.0645(6) \\ \mathrm{H} 20 & 0.0410 & -0.1687 & 0.3352 & 0.077^{*} \\ \mathrm{C} 21 & 0.2574(3) & -0.1733(2) & 0.2569(2) & 0.0614(6) \\ \mathrm{H} 21 & 0.2796 & -0.2575 & 0.3017 & 0.074^{*} \\ \mathrm{C} 22 & 0.3509(2) & -0.0813(2) & 0.1635(2) & 0.0572(5) \\ \mathrm{H} 22 & 0.4502 & -0.0900 & 0.1316 & 0.069^{*} \\ \mathrm{C} 11 & 0.79773(17) & 0.27959(16) & 0.21235(16) & 0.0371(4) \\ \mathrm{C} 12 & 0.8079(3) & 0.1308(2) & 0.0852(2) & 0.0628(6) \\ \mathrm{H} 12 \mathrm{~A} & 0.7741 & 0.1816 & 0.0171 & 0.094^{*} \\ \mathrm{H} 12 \mathrm{~B} & 0.7322 & 0.0844 & 0.1536 & 0.094^{*} \\ \mathrm{H} 12 \mathrm{C} & 0.8707 & 0.0716 & 0.0512 & 0.094^{*} \\ \mathrm{C} 23 & 0.29892(18) & 0.35980(17) & 0.21196(17) & 0.0384(4) \\ \mathrm{C} 24 & 0.3153(3) & 0.5670(2) & 0.0756(2) & 0.0686(6) \\ \mathrm{H} 24 \mathrm{~A} & 0.3801 & 0.6390 & 0.0357 & 0.103^{*} \\ \mathrm{H} 24 \mathrm{~B} & 0.2388 & 0.5897 & 0.1386 & 0.103^{*} \\ \mathrm{H} 24 \mathrm{C} & 0.2831 & 0.5424 & 0.0103 & 0.103^{*} \\ \mathrm{~N} 1 & 0.87534(16) & 0.34529(15) & 0.25925(16) & 0.0418(3) \\ \mathrm{N} 2 & 0.37378(16) & 0.27173(15) & 0.26751(16) & 0.0414(3) \\ \mathrm{O} 1 & 0.67396(13) & 0.27931(14) & 0.23547(14) & 0.0507(3) \\ \mathrm{O} 2 & 0.87800(14) & 0.21268(14) & 0.13527(14) & 0.0526(3) \\ \mathrm{O} 3 & 0.17679(13) & 0.35027(14) & 0.22624(15) & 0.0551(4) \\ \mathrm{O} 4 & 0.38073(14) & 0.46244(13) & 0.13837(14) & 0.0556(4) \\ \mathrm{H} 2 & 0.455(2) & 0.284(2) & 0.248(2) & 0.049(6)^{*} \\ \mathrm{H} 1 & 0.959(2) & 0.3413(19) & 0.2356(19) & 0.044(6)^{*} \\ & & & & \end{array}$

Atomic displacement parameters $\left(\AA^{2}\right)$

\begin{tabular}{lllllll}
\hline & $U^{11}$ & $U^{22}$ & $U^{33}$ & $U^{12}$ & $U^{13}$ & $U^{23}$ \\
\hline Fe1 & $0.03477(14)$ & $0.03807(14)$ & $0.04083(14)$ & $0.00500(10)$ & $-0.01523(11)$ & $-0.00900(10)$ \\
Fe2 & $0.03208(13)$ & $0.04001(14)$ & $0.03383(13)$ & $0.00118(10)$ & $-0.01287(10)$ & $-0.01047(10)$ \\
C1 & $0.0358(9)$ & $0.0422(9)$ & $0.0416(9)$ & $0.0111(7)$ & $-0.0151(7)$ & $-0.0113(7)$ \\
C2 & $0.0422(10)$ & $0.0412(10)$ & $0.0439(10)$ & $0.0078(8)$ & $-0.0052(8)$ & $-0.0026(8)$ \\
C3 & $0.0632(13)$ & $0.0574(12)$ & $0.0382(10)$ & $0.0231(10)$ & $-0.0092(9)$ & $-0.0096(9)$ \\
C4 & $0.0704(14)$ & $0.0631(13)$ & $0.0521(12)$ & $0.0266(11)$ & $-0.0359(11)$ & $-0.0270(10)$ \\
C5 & $0.0429(10)$ & $0.0597(12)$ & $0.0564(12)$ & $0.0164(9)$ & $-0.0290(9)$ & $-0.0234(10)$ \\
C6 & $0.0788(17)$ & $0.0598(14)$ & $0.0917(19)$ & $-0.0075(12)$ & $-0.0635(16)$ & $0.0112(13)$ \\
C7 & $0.0659(16)$ & $0.0719(17)$ & $0.0765(17)$ & $0.0334(13)$ & $-0.0146(13)$ & $0.0105(14)$ \\
C8 & $0.122(2)$ & $0.0374(11)$ & $0.0737(17)$ & $0.0061(13)$ & $-0.0441(17)$ & $-0.0096(11)$ \\
C9 & $0.0667(15)$ & $0.0652(15)$ & $0.0657(15)$ & $-0.0199(12)$ & $-0.0221(12)$ & $0.0082(12)$ \\
C10 & $0.096(2)$ & $0.0683(15)$ & $0.0440(12)$ & $0.0130(13)$ & $-0.0288(12)$ & $-0.0086(11)$ \\
C13 & $0.0331(8)$ & $0.0445(9)$ & $0.0362(9)$ & $-0.0047(7)$ & $-0.0128(7)$ & $-0.0103(7)$ \\
C14 & $0.0364(9)$ & $0.0516(11)$ & $0.0406(9)$ & $-0.0068(8)$ & $-0.0043(7)$ & $-0.0205(8)$ \\
C15 & $0.0548(12)$ & $0.0611(12)$ & $0.0327(9)$ & $-0.0195(10)$ & $-0.0064(8)$ & $-0.0114(8)$ \\
C16 & $0.0630(13)$ & $0.0494(11)$ & $0.0414(10)$ & $-0.0100(9)$ & $-0.0291(9)$ & $0.0004(8)$ \\
C17 & $0.0376(9)$ & $0.0510(11)$ & $0.0462(10)$ & $-0.0033(8)$ & $-0.0229(8)$ & $-0.0050(8)$ \\
C18 & $0.0816(16)$ & $0.0578(13)$ & $0.0376(10)$ & $0.0065(11)$ & $-0.0251(10)$ & $-0.0140(9)$ \\
C19 & $0.0644(14)$ & $0.0859(17)$ & $0.0729(15)$ & $0.0335(12)$ & $-0.0479(12)$ & $-0.0507(14)$ \\
C20 & $0.0566(13)$ & $0.0851(17)$ & $0.0631(14)$ & $-0.0173(12)$ & $-0.0159(11)$ & $-0.0393(13)$
\end{tabular}




\begin{tabular}{lllllll}
$\mathrm{C} 21$ & $0.0897(17)$ & $0.0461(11)$ & $0.0613(13)$ & $0.0114(11)$ & $-0.0329(13)$ & $-0.0242(10)$ \\
$\mathrm{C} 22$ & $0.0531(12)$ & $0.0751(15)$ & $0.0506(12)$ & $0.0185(11)$ & $-0.0139(10)$ & $-0.0304(11)$ \\
$\mathrm{C} 11$ & $0.0352(9)$ & $0.0367(9)$ & $0.0373(9)$ & $0.0032(7)$ & $-0.0123(7)$ & $-0.0029(7)$ \\
$\mathrm{C} 12$ & $0.0772(16)$ & $0.0549(13)$ & $0.0674(14)$ & $0.0007(11)$ & $-0.0290(12)$ & $-0.0256(11)$ \\
$\mathrm{C} 23$ & $0.0358(9)$ & $0.0428(9)$ & $0.0402(9)$ & $0.0051(7)$ & $-0.0126(7)$ & $-0.0148(7)$ \\
$\mathrm{C} 24$ & $0.0804(17)$ & $0.0506(13)$ & $0.0726(15)$ & $0.0156(12)$ & $-0.0287(13)$ & $-0.0035(11)$ \\
$\mathrm{N} 1$ & $0.0265(7)$ & $0.0508(9)$ & $0.0540(9)$ & $0.0074(6)$ & $-0.0129(7)$ & $-0.0226(7)$ \\
$\mathrm{N} 2$ & $0.0256(7)$ & $0.0432(8)$ & $0.0529(9)$ & $-0.0026(6)$ & $-0.0125(7)$ & $-0.0048(7)$ \\
$\mathrm{O} 1$ & $0.0323(7)$ & $0.0616(9)$ & $0.0608(8)$ & $0.0011(6)$ & $-0.0182(6)$ & $-0.0139(7)$ \\
$\mathrm{O} 2$ & $0.0460(8)$ & $0.0602(9)$ & $0.0608(9)$ & $0.0062(6)$ & $-0.0160(7)$ & $-0.0313(7)$ \\
$\mathrm{O} 3$ & $0.0335(7)$ & $0.0634(9)$ & $0.0714(10)$ & $0.0089(6)$ & $-0.0211(7)$ & $-0.0153(7)$ \\
$\mathrm{O} 4$ & $0.0485(8)$ & $0.0472(8)$ & $0.0623(9)$ & $0.0024(6)$ & $-0.0161(7)$ & $0.0039(7)$ \\
\hline
\end{tabular}

Geometric parameters $\left(\AA,{ }^{\circ}\right)$

\begin{tabular}{|c|c|c|c|}
\hline $\mathrm{Fe} 1-\mathrm{C} 7$ & $2.023(2)$ & C9-H9 & 0.9800 \\
\hline $\mathrm{Fe} 1-\mathrm{C} 6$ & $2.027(2)$ & $\mathrm{C} 10-\mathrm{H} 10$ & 0.9800 \\
\hline $\mathrm{Fe} 1-\mathrm{C} 4$ & $2.0296(19)$ & $\mathrm{C} 13-\mathrm{N} 2$ & $1.407(2)$ \\
\hline $\mathrm{Fe} 1-\mathrm{C} 8$ & $2.030(2)$ & $\mathrm{C} 13-\mathrm{C} 17$ & $1.414(3)$ \\
\hline $\mathrm{Fe} 1-\mathrm{C} 9$ & $2.034(2)$ & $\mathrm{C} 13-\mathrm{C} 14$ & $1.422(2)$ \\
\hline $\mathrm{Fe} 1-\mathrm{C} 10$ & $2.035(2)$ & $\mathrm{C} 14-\mathrm{C} 15$ & $1.413(3)$ \\
\hline $\mathrm{Fe} 1-\mathrm{C} 3$ & $2.037(2)$ & $\mathrm{C} 14-\mathrm{H} 14$ & 0.9800 \\
\hline $\mathrm{Fe} 1-\mathrm{C} 5$ & $2.0410(18)$ & $\mathrm{C} 15-\mathrm{C} 16$ & $1.413(3)$ \\
\hline $\mathrm{Fe} 1-\mathrm{C} 2$ & $2.0484(19)$ & C15-H15 & 0.9800 \\
\hline $\mathrm{Fe} 1-\mathrm{C} 1$ & $2.0571(17)$ & $\mathrm{C} 16-\mathrm{C} 17$ & $1.420(3)$ \\
\hline $\mathrm{Fe} 2-\mathrm{C} 19$ & $2.0292(19)$ & $\mathrm{C} 16-\mathrm{H} 16$ & 0.9800 \\
\hline $\mathrm{Fe} 2-\mathrm{C} 18$ & 2.0319 (19) & $\mathrm{C} 17-\mathrm{H} 17$ & 0.9800 \\
\hline $\mathrm{Fe} 2-\mathrm{C} 22$ & $2.032(2)$ & $\mathrm{C} 18-\mathrm{C} 22$ & $1.401(3)$ \\
\hline $\mathrm{Fe} 2-\mathrm{C} 16$ & $2.0336(18)$ & $\mathrm{C} 18-\mathrm{C} 19$ & $1.405(3)$ \\
\hline $\mathrm{Fe} 2-\mathrm{C} 20$ & $2.034(2)$ & $\mathrm{C} 18-\mathrm{H} 18$ & 0.9800 \\
\hline $\mathrm{Fe} 2-\mathrm{C} 21$ & $2.034(2)$ & $\mathrm{C} 19-\mathrm{C} 20$ & $1.409(4)$ \\
\hline $\mathrm{Fe} 2-\mathrm{C} 15$ & $2.0352(18)$ & $\mathrm{C} 19-\mathrm{H} 19$ & 0.9800 \\
\hline $\mathrm{Fe} 2-\mathrm{C} 17$ & $2.0394(17)$ & $\mathrm{C} 20-\mathrm{C} 21$ & $1.396(3)$ \\
\hline $\mathrm{Fe} 2-\mathrm{C} 14$ & $2.0447(18)$ & $\mathrm{C} 20-\mathrm{H} 20$ & 0.9800 \\
\hline $\mathrm{Fe} 2-\mathrm{C} 13$ & $2.0500(17)$ & $\mathrm{C} 21-\mathrm{C} 22$ & $1.395(3)$ \\
\hline $\mathrm{C} 1-\mathrm{N} 1$ & $1.404(2)$ & $\mathrm{C} 21-\mathrm{H} 21$ & 0.9800 \\
\hline $\mathrm{C} 1-\mathrm{C} 5$ & $1.418(3)$ & $\mathrm{C} 22-\mathrm{H} 22$ & 0.9800 \\
\hline $\mathrm{C} 1-\mathrm{C} 2$ & $1.422(2)$ & $\mathrm{C} 11-\mathrm{O} 1$ & $1.207(2)$ \\
\hline $\mathrm{C} 2-\mathrm{C} 3$ & $1.416(3)$ & $\mathrm{C} 11-\mathrm{O} 2$ & $1.341(2)$ \\
\hline $\mathrm{C} 2-\mathrm{H} 2 \mathrm{~A}$ & 0.9800 & $\mathrm{C} 11-\mathrm{N} 1$ & $1.344(2)$ \\
\hline $\mathrm{C} 3-\mathrm{C} 4$ & $1.407(3)$ & $\mathrm{C} 12-\mathrm{O} 2$ & $1.435(2)$ \\
\hline $\mathrm{C} 3-\mathrm{H} 3$ & 0.9800 & $\mathrm{C} 12-\mathrm{H} 12 \mathrm{~A}$ & 0.9600 \\
\hline $\mathrm{C} 4-\mathrm{C} 5$ & $1.420(3)$ & $\mathrm{C} 12-\mathrm{H} 12 \mathrm{~B}$ & 0.9600 \\
\hline $\mathrm{C} 4-\mathrm{H} 4$ & 0.9800 & $\mathrm{C} 12-\mathrm{H} 12 \mathrm{C}$ & 0.9600 \\
\hline $\mathrm{C} 5-\mathrm{H} 5$ & 0.9800 & $\mathrm{C} 23-\mathrm{O} 3$ & $1.205(2)$ \\
\hline $\mathrm{C} 6-\mathrm{C} 10$ & $1.395(4)$ & $\mathrm{C} 23-\mathrm{O} 4$ & $1.341(2)$ \\
\hline $\mathrm{C} 6-\mathrm{C} 7$ & $1.409(4)$ & $\mathrm{C} 23-\mathrm{N} 2$ & $1.344(2)$ \\
\hline $\mathrm{C} 6-\mathrm{H} 6$ & 0.9800 & $\mathrm{C} 24-\mathrm{O} 4$ & $1.430(3)$ \\
\hline
\end{tabular}




\begin{tabular}{|c|c|c|c|}
\hline $\mathrm{C} 7-\mathrm{C} 8$ & $1.393(4)$ & $\mathrm{C} 24-\mathrm{H} 24 \mathrm{~A}$ & 0.9600 \\
\hline $\mathrm{C} 7-\mathrm{H} 7$ & 0.9800 & $\mathrm{C} 24-\mathrm{H} 24 \mathrm{~B}$ & 0.9600 \\
\hline $\mathrm{C} 8-\mathrm{C} 9$ & $1.379(4)$ & $\mathrm{C} 24-\mathrm{H} 24 \mathrm{C}$ & 0.9600 \\
\hline $\mathrm{C} 8-\mathrm{H} 8$ & 0.9800 & $\mathrm{~N} 1-\mathrm{H} 1$ & $0.82(2)$ \\
\hline $\mathrm{C} 9-\mathrm{C} 10$ & $1.386(3)$ & $\mathrm{N} 2-\mathrm{H} 2$ & $0.80(2)$ \\
\hline $\mathrm{C} 7-\mathrm{Fe} 1-\mathrm{C} 6$ & $40.72(12)$ & $\mathrm{C} 4-\mathrm{C} 5-\mathrm{H} 5$ & 126.2 \\
\hline $\mathrm{C} 7-\mathrm{Fe} 1-\mathrm{C} 4$ & $126.60(11)$ & $\mathrm{Fe} 1-\mathrm{C} 5-\mathrm{H} 5$ & 126.2 \\
\hline $\mathrm{C} 6-\mathrm{Fe} 1-\mathrm{C} 4$ & $165.02(12)$ & $\mathrm{C} 10-\mathrm{C} 6-\mathrm{C} 7$ & $107.6(2)$ \\
\hline $\mathrm{C} 7-\mathrm{Fe} 1-\mathrm{C} 8$ & $40.21(12)$ & $\mathrm{C} 10-\mathrm{C} 6-\mathrm{Fe} 1$ & $70.21(13)$ \\
\hline $\mathrm{C} 6-\mathrm{Fe} 1-\mathrm{C} 8$ & $67.83(11)$ & $\mathrm{C} 7-\mathrm{C} 6-\mathrm{Fe} 1$ & $69.50(13)$ \\
\hline $\mathrm{C} 4-\mathrm{Fe} 1-\mathrm{C} 8$ & $107.38(10)$ & $\mathrm{C} 10-\mathrm{C} 6-\mathrm{H} 6$ & 126.2 \\
\hline $\mathrm{C} 7-\mathrm{Fe} 1-\mathrm{C} 9$ & $67.02(11)$ & $\mathrm{C} 7-\mathrm{C} 6-\mathrm{H} 6$ & 126.2 \\
\hline $\mathrm{C} 6-\mathrm{Fe} 1-\mathrm{C} 9$ & $67.10(10)$ & $\mathrm{Fe} 1-\mathrm{C} 6-\mathrm{H} 6$ & 126.2 \\
\hline $\mathrm{C} 4-\mathrm{Fe} 1-\mathrm{C} 9$ & $118.98(10)$ & $\mathrm{C} 8-\mathrm{C} 7-\mathrm{C} 6$ & $107.7(2)$ \\
\hline $\mathrm{C} 8-\mathrm{Fe} 1-\mathrm{C} 9$ & 39.67 (11) & $\mathrm{C} 8-\mathrm{C} 7-\mathrm{Fe} 1$ & $70.14(14)$ \\
\hline $\mathrm{C} 7-\mathrm{Fe} 1-\mathrm{C} 10$ & $67.77(11)$ & $\mathrm{C} 6-\mathrm{C} 7-\mathrm{Fe} 1$ & $69.79(13)$ \\
\hline $\mathrm{C} 6-\mathrm{Fe} 1-\mathrm{C} 10$ & $40.17(11)$ & $\mathrm{C} 8-\mathrm{C} 7-\mathrm{H} 7$ & 126.1 \\
\hline $\mathrm{C} 4-\mathrm{Fe} 1-\mathrm{C} 10$ & $152.75(11)$ & $\mathrm{C} 6-\mathrm{C} 7-\mathrm{H} 7$ & 126.1 \\
\hline $\mathrm{C} 8-\mathrm{Fe} 1-\mathrm{C} 10$ & $67.40(11)$ & $\mathrm{Fe} 1-\mathrm{C} 7-\mathrm{H} 7$ & 126.1 \\
\hline $\mathrm{C} 9-\mathrm{Fe} 1-\mathrm{C} 10$ & $39.84(10)$ & $\mathrm{C} 9-\mathrm{C} 8-\mathrm{C} 7$ & $107.8(2)$ \\
\hline $\mathrm{C} 7-\mathrm{Fe} 1-\mathrm{C} 3$ & $108.40(10)$ & $\mathrm{C} 9-\mathrm{C} 8-\mathrm{Fe} 1$ & $70.32(14)$ \\
\hline $\mathrm{C} 6-\mathrm{Fe} 1-\mathrm{C} 3$ & $128.18(11)$ & $\mathrm{C} 7-\mathrm{C} 8-\mathrm{Fe} 1$ & 69.64 (13) \\
\hline $\mathrm{C} 4-\mathrm{Fe} 1-\mathrm{C} 3$ & $40.48(9)$ & $\mathrm{C} 9-\mathrm{C} 8-\mathrm{H} 8$ & 126.1 \\
\hline $\mathrm{C} 8-\mathrm{Fe} 1-\mathrm{C} 3$ & $119.16(10)$ & $\mathrm{C} 7-\mathrm{C} 8-\mathrm{H} 8$ & 126.1 \\
\hline $\mathrm{C} 9-\mathrm{Fe} 1-\mathrm{C} 3$ & $152.71(10)$ & $\mathrm{Fe} 1-\mathrm{C} 8-\mathrm{H} 8$ & 126.1 \\
\hline $\mathrm{C} 10-\mathrm{Fe} 1-\mathrm{C} 3$ & $165.88(11)$ & $\mathrm{C} 8-\mathrm{C} 9-\mathrm{C} 10$ & $109.3(2)$ \\
\hline $\mathrm{C} 7-\mathrm{Fe} 1-\mathrm{C} 5$ & $163.91(12)$ & $\mathrm{C} 8-\mathrm{C} 9-\mathrm{Fe} 1$ & $70.01(14)$ \\
\hline $\mathrm{C} 6-\mathrm{Fe} 1-\mathrm{C} 5$ & $153.42(11)$ & $\mathrm{C} 10-\mathrm{C} 9-\mathrm{Fe} 1$ & $70.12(13)$ \\
\hline $\mathrm{C} 4-\mathrm{Fe} 1-\mathrm{C} 5$ & $40.82(8)$ & $\mathrm{C} 8-\mathrm{C} 9-\mathrm{H} 9$ & 125.4 \\
\hline $\mathrm{C} 8-\mathrm{Fe} 1-\mathrm{C} 5$ & $126.38(11)$ & $\mathrm{C} 10-\mathrm{C} 9-\mathrm{H} 9$ & 125.4 \\
\hline $\mathrm{C} 9-\mathrm{Fe} 1-\mathrm{C} 5$ & $108.18(10)$ & $\mathrm{Fe} 1-\mathrm{C} 9-\mathrm{H} 9$ & 125.4 \\
\hline $\mathrm{C} 10-\mathrm{Fe} 1-\mathrm{C} 5$ & $119.19(10)$ & $\mathrm{C} 9-\mathrm{C} 10-\mathrm{C} 6$ & $107.6(2)$ \\
\hline $\mathrm{C} 3-\mathrm{Fe} 1-\mathrm{C} 5$ & $68.34(9)$ & $\mathrm{C} 9-\mathrm{C} 10-\mathrm{Fe} 1$ & $70.04(13)$ \\
\hline $\mathrm{C} 7-\mathrm{Fe} 1-\mathrm{C} 2$ & $120.04(10)$ & $\mathrm{C} 6-\mathrm{C} 10-\mathrm{Fe} 1$ & $69.62(13)$ \\
\hline $\mathrm{C} 6-\mathrm{Fe} 1-\mathrm{C} 2$ & $109.18(9)$ & $\mathrm{C} 9-\mathrm{C} 10-\mathrm{H} 10$ & 126.2 \\
\hline $\mathrm{C} 4-\mathrm{Fe} 1-\mathrm{C} 2$ & $68.36(9)$ & $\mathrm{C} 6-\mathrm{C} 10-\mathrm{H} 10$ & 126.2 \\
\hline $\mathrm{C} 8-\mathrm{Fe} 1-\mathrm{C} 2$ & $153.54(11)$ & $\mathrm{Fe} 1-\mathrm{C} 10-\mathrm{H} 10$ & 126.2 \\
\hline $\mathrm{C} 9-\mathrm{Fe} 1-\mathrm{C} 2$ & $165.55(10)$ & $\mathrm{N} 2-\mathrm{C} 13-\mathrm{C} 17$ & $123.13(16)$ \\
\hline $\mathrm{C} 10-\mathrm{Fe} 1-\mathrm{C} 2$ & $128.38(10)$ & $\mathrm{N} 2-\mathrm{C} 13-\mathrm{C} 14$ & $128.28(17)$ \\
\hline $\mathrm{C} 3-\mathrm{Fe} 1-\mathrm{C} 2$ & $40.57(8)$ & $\mathrm{C} 17-\mathrm{C} 13-\mathrm{C} 14$ & $108.49(16)$ \\
\hline $\mathrm{C} 5-\mathrm{Fe} 1-\mathrm{C} 2$ & $68.48(8)$ & $\mathrm{N} 2-\mathrm{C} 13-\mathrm{Fe} 2$ & $129.73(12)$ \\
\hline $\mathrm{C} 7-\mathrm{Fe} 1-\mathrm{C} 1$ & $154.45(11)$ & $\mathrm{C} 17-\mathrm{C} 13-\mathrm{Fe} 2$ & $69.37(10)$ \\
\hline $\mathrm{C} 6-\mathrm{Fe} 1-\mathrm{C} 1$ & $120.32(10)$ & $\mathrm{C} 14-\mathrm{C} 13-\mathrm{Fe} 2$ & $69.48(10)$ \\
\hline $\mathrm{C} 4-\mathrm{Fe} 1-\mathrm{C} 1$ & $68.13(8)$ & $\mathrm{C} 15-\mathrm{C} 14-\mathrm{C} 13$ & $107.36(17)$ \\
\hline $\mathrm{C} 8-\mathrm{Fe} 1-\mathrm{C} 1$ & $164.23(11)$ & $\mathrm{C} 15-\mathrm{C} 14-\mathrm{Fe} 2$ & $69.38(11)$ \\
\hline $\mathrm{C} 9-\mathrm{Fe} 1-\mathrm{C} 1$ & $128.00(10)$ & $\mathrm{C} 13-\mathrm{C} 14-\mathrm{Fe} 2$ & $69.89(10)$ \\
\hline $\mathrm{C} 10-\mathrm{Fe} 1-\mathrm{C} 1$ & $109.21(9)$ & $\mathrm{C} 15-\mathrm{C} 14-\mathrm{H} 14$ & 126.3 \\
\hline
\end{tabular}




\begin{tabular}{|c|c|c|c|}
\hline $\mathrm{C} 3-\mathrm{Fe} 1-\mathrm{C} 1$ & $67.97(8)$ & $\mathrm{C} 13-\mathrm{C} 14-\mathrm{H} 14$ & 126.3 \\
\hline $\mathrm{C} 5-\mathrm{Fe} 1-\mathrm{C} 1$ & $40.48(7)$ & $\mathrm{Fe} 2-\mathrm{C} 14-\mathrm{H} 14$ & 126.3 \\
\hline $\mathrm{C} 2-\mathrm{Fe} 1-\mathrm{C} 1$ & $40.54(7)$ & $\mathrm{C} 16-\mathrm{C} 15-\mathrm{C} 14$ & $108.53(17)$ \\
\hline $\mathrm{C} 19-\mathrm{Fe} 2-\mathrm{C} 18$ & $40.49(10)$ & $\mathrm{C} 16-\mathrm{C} 15-\mathrm{Fe} 2$ & $69.62(11)$ \\
\hline $\mathrm{C} 19-\mathrm{Fe} 2-\mathrm{C} 22$ & $67.75(9)$ & $\mathrm{C} 14-\mathrm{C} 15-\mathrm{Fe} 2$ & $70.10(10)$ \\
\hline $\mathrm{C} 18-\mathrm{Fe} 2-\mathrm{C} 22$ & $40.34(9)$ & $\mathrm{C} 16-\mathrm{C} 15-\mathrm{H} 15$ & 125.7 \\
\hline $\mathrm{C} 19-\mathrm{Fe} 2-\mathrm{C} 16$ & $162.31(10)$ & $\mathrm{C} 14-\mathrm{C} 15-\mathrm{H} 15$ & 125.7 \\
\hline $\mathrm{C} 18-\mathrm{Fe} 2-\mathrm{C} 16$ & $155.74(9)$ & $\mathrm{Fe} 2-\mathrm{C} 15-\mathrm{H} 15$ & 125.7 \\
\hline $\mathrm{C} 22-\mathrm{Fe} 2-\mathrm{C} 16$ & $121.11(9)$ & $\mathrm{C} 15-\mathrm{C} 16-\mathrm{C} 17$ & $108.03(18)$ \\
\hline $\mathrm{C} 19-\mathrm{Fe} 2-\mathrm{C} 20$ & $40.57(10)$ & $\mathrm{C} 15-\mathrm{C} 16-\mathrm{Fe} 2$ & $69.75(11)$ \\
\hline $\mathrm{C} 18-\mathrm{Fe} 2-\mathrm{C} 20$ & $68.05(10)$ & $\mathrm{C} 17-\mathrm{C} 16-\mathrm{Fe} 2$ & $69.82(10)$ \\
\hline $\mathrm{C} 22-\mathrm{Fe} 2-\mathrm{C} 20$ & $67.53(9)$ & $\mathrm{C} 15-\mathrm{C} 16-\mathrm{H} 16$ & 126.0 \\
\hline $\mathrm{C} 16-\mathrm{Fe} 2-\mathrm{C} 20$ & $125.34(10)$ & $\mathrm{C} 17-\mathrm{C} 16-\mathrm{H} 16$ & 126.0 \\
\hline $\mathrm{C} 19-\mathrm{Fe} 2-\mathrm{C} 21$ & $67.83(9)$ & $\mathrm{Fe} 2-\mathrm{C} 16-\mathrm{H} 16$ & 126.0 \\
\hline $\mathrm{C} 18-\mathrm{Fe} 2-\mathrm{C} 21$ & $67.84(9)$ & $\mathrm{C} 13-\mathrm{C} 17-\mathrm{C} 16$ & $107.58(17)$ \\
\hline $\mathrm{C} 22-\mathrm{Fe} 2-\mathrm{C} 21$ & $40.11(10)$ & $\mathrm{C} 13-\mathrm{C} 17-\mathrm{Fe} 2$ & $70.18(9)$ \\
\hline $\mathrm{C} 16-\mathrm{Fe} 2-\mathrm{C} 21$ & $108.15(9)$ & $\mathrm{C} 16-\mathrm{C} 17-\mathrm{Fe} 2$ & $69.38(10)$ \\
\hline $\mathrm{C} 20-\mathrm{Fe} 2-\mathrm{C} 21$ & $40.13(10)$ & $\mathrm{C} 13-\mathrm{C} 17-\mathrm{H} 17$ & 126.2 \\
\hline $\mathrm{C} 19-\mathrm{Fe} 2-\mathrm{C} 15$ & $125.56(9)$ & $\mathrm{C} 16-\mathrm{C} 17-\mathrm{H} 17$ & 126.2 \\
\hline $\mathrm{C} 18-\mathrm{Fe} 2-\mathrm{C} 15$ & $162.01(9)$ & $\mathrm{Fe} 2-\mathrm{C} 17-\mathrm{H} 17$ & 126.2 \\
\hline $\mathrm{C} 22-\mathrm{Fe} 2-\mathrm{C} 15$ & $156.46(9)$ & $\mathrm{C} 22-\mathrm{C} 18-\mathrm{C} 19$ & $107.5(2)$ \\
\hline $\mathrm{C} 16-\mathrm{Fe} 2-\mathrm{C} 15$ & $40.63(9)$ & $\mathrm{C} 22-\mathrm{C} 18-\mathrm{Fe} 2$ & $69.84(12)$ \\
\hline $\mathrm{C} 20-\mathrm{Fe} 2-\mathrm{C} 15$ & $108.67(9)$ & $\mathrm{C} 19-\mathrm{C} 18-\mathrm{Fe} 2$ & $69.65(12)$ \\
\hline $\mathrm{C} 21-\mathrm{Fe} 2-\mathrm{C} 15$ & $121.89(9)$ & $\mathrm{C} 22-\mathrm{C} 18-\mathrm{H} 18$ & 126.2 \\
\hline $\mathrm{C} 19-\mathrm{Fe} 2-\mathrm{C} 17$ & $155.73(10)$ & $\mathrm{C} 19-\mathrm{C} 18-\mathrm{H} 18$ & 126.2 \\
\hline $\mathrm{C} 18-\mathrm{Fe} 2-\mathrm{C} 17$ & $120.36(9)$ & $\mathrm{Fe} 2-\mathrm{C} 18-\mathrm{H} 18$ & 126.2 \\
\hline $\mathrm{C} 22-\mathrm{Fe} 2-\mathrm{C} 17$ & $107.46(9)$ & $\mathrm{C} 18-\mathrm{C} 19-\mathrm{C} 20$ & $107.9(2)$ \\
\hline $\mathrm{C} 16-\mathrm{Fe} 2-\mathrm{C} 17$ & $40.80(7)$ & $\mathrm{C} 18-\mathrm{C} 19-\mathrm{Fe} 2$ & $69.86(11)$ \\
\hline $\mathrm{C} 20-\mathrm{Fe} 2-\mathrm{C} 17$ & $161.74(10)$ & $\mathrm{C} 20-\mathrm{C} 19-\mathrm{Fe} 2$ & $69.90(12)$ \\
\hline $\mathrm{C} 21-\mathrm{Fe} 2-\mathrm{C} 17$ & $124.84(9)$ & $\mathrm{C} 18-\mathrm{C} 19-\mathrm{H} 19$ & 126.1 \\
\hline $\mathrm{C} 15-\mathrm{Fe} 2-\mathrm{C} 17$ & $68.45(8)$ & $\mathrm{C} 20-\mathrm{C} 19-\mathrm{H} 19$ & 126.1 \\
\hline $\mathrm{C} 19-\mathrm{Fe} 2-\mathrm{C} 14$ & $108.02(8)$ & $\mathrm{Fe} 2-\mathrm{C} 19-\mathrm{H} 19$ & 126.1 \\
\hline $\mathrm{C} 18-\mathrm{Fe} 2-\mathrm{C} 14$ & $124.87(9)$ & $\mathrm{C} 21-\mathrm{C} 20-\mathrm{C} 19$ & $107.9(2)$ \\
\hline $\mathrm{C} 22-\mathrm{Fe} 2-\mathrm{C} 14$ & $161.60(9)$ & $\mathrm{C} 21-\mathrm{C} 20-\mathrm{Fe} 2$ & $69.95(12)$ \\
\hline $\mathrm{C} 16-\mathrm{Fe} 2-\mathrm{C} 14$ & $68.44(9)$ & $\mathrm{C} 19-\mathrm{C} 20-\mathrm{Fe} 2$ & $69.53(12)$ \\
\hline $\mathrm{C} 20-\mathrm{Fe} 2-\mathrm{C} 14$ & $121.77(9)$ & $\mathrm{C} 21-\mathrm{C} 20-\mathrm{H} 20$ & 126.1 \\
\hline $\mathrm{C} 21-\mathrm{Fe} 2-\mathrm{C} 14$ & $156.75(9)$ & $\mathrm{C} 19-\mathrm{C} 20-\mathrm{H} 20$ & 126.1 \\
\hline $\mathrm{C} 15-\mathrm{Fe} 2-\mathrm{C} 14$ & $40.53(8)$ & $\mathrm{Fe} 2-\mathrm{C} 20-\mathrm{H} 20$ & 126.1 \\
\hline $\mathrm{C} 17-\mathrm{Fe} 2-\mathrm{C} 14$ & $68.59(8)$ & $\mathrm{C} 22-\mathrm{C} 21-\mathrm{C} 20$ & $108.2(2)$ \\
\hline $\mathrm{C} 19-\mathrm{Fe} 2-\mathrm{C} 13$ & $121.31(9)$ & $\mathrm{C} 22-\mathrm{C} 21-\mathrm{Fe} 2$ & $69.85(12)$ \\
\hline $\mathrm{C} 18-\mathrm{Fe} 2-\mathrm{C} 13$ & $107.63(8)$ & $\mathrm{C} 20-\mathrm{C} 21-\mathrm{Fe} 2$ & $69.92(12)$ \\
\hline $\mathrm{C} 22-\mathrm{Fe} 2-\mathrm{C} 13$ & $124.88(9)$ & $\mathrm{C} 22-\mathrm{C} 21-\mathrm{H} 21$ & 125.9 \\
\hline $\mathrm{C} 16-\mathrm{Fe} 2-\mathrm{C} 13$ & $68.09(7)$ & $\mathrm{C} 20-\mathrm{C} 21-\mathrm{H} 21$ & 125.9 \\
\hline $\mathrm{C} 20-\mathrm{Fe} 2-\mathrm{C} 13$ & $156.81(9)$ & $\mathrm{Fe} 2-\mathrm{C} 21-\mathrm{H} 21$ & 125.9 \\
\hline $\mathrm{C} 21-\mathrm{Fe} 2-\mathrm{C} 13$ & $161.39(9)$ & $\mathrm{C} 21-\mathrm{C} 22-\mathrm{C} 18$ & $108.5(2)$ \\
\hline $\mathrm{C} 15-\mathrm{Fe} 2-\mathrm{C} 13$ & $67.98(7)$ & $\mathrm{C} 21-\mathrm{C} 22-\mathrm{Fe} 2$ & $70.04(12)$ \\
\hline $\mathrm{C} 17-\mathrm{Fe} 2-\mathrm{C} 13$ & $40.46(7)$ & $\mathrm{C} 18-\mathrm{C} 22-\mathrm{Fe} 2$ & $69.82(12)$ \\
\hline $\mathrm{C} 14-\mathrm{Fe} 2-\mathrm{C} 13$ & $40.63(7)$ & $\mathrm{C} 21-\mathrm{C} 22-\mathrm{H} 22$ & 125.7 \\
\hline
\end{tabular}




$\begin{array}{llll}\mathrm{N} 1-\mathrm{C} 1-\mathrm{C} 5 & 123.16(16) & \mathrm{C} 18-\mathrm{C} 22-\mathrm{H} 22 & 125.7 \\ \mathrm{~N} 1-\mathrm{C} 1-\mathrm{C} 2 & 128.49(17) & \mathrm{Fe} 2-\mathrm{C} 22-\mathrm{H} 22 & 125.7 \\ \mathrm{C} 5-\mathrm{C} 1-\mathrm{C} 2 & 108.21(17) & \mathrm{O} 1-\mathrm{C} 11-\mathrm{O} 2 & 124.19(17) \\ \mathrm{N} 1-\mathrm{C} 1-\mathrm{Fe} 1 & 130.32(13) & \mathrm{O} 1-\mathrm{C} 11-\mathrm{N} 1 & 125.81(17) \\ \mathrm{C} 5-\mathrm{C} 1-\mathrm{Fe} 1 & 69.15(10) & \mathrm{O} 2-\mathrm{C} 11-\mathrm{N} 1 & 109.99(15) \\ \mathrm{C} 2-\mathrm{C} 1-\mathrm{Fe} 1 & 69.40(10) & \mathrm{O} 2-\mathrm{C} 12-\mathrm{H} 12 \mathrm{~A} & 109.5 \\ \mathrm{C} 3-\mathrm{C} 2-\mathrm{C} 1 & 107.45(18) & \mathrm{O} 2-\mathrm{C} 12-\mathrm{H} 12 \mathrm{~B} & 109.5 \\ \mathrm{C} 3-\mathrm{C} 2-\mathrm{Fe} 1 & 69.28(11) & \mathrm{H} 12 \mathrm{~A}-\mathrm{C} 12-\mathrm{H} 12 \mathrm{~B} & 109.5 \\ \mathrm{C} 1-\mathrm{C} 2-\mathrm{Fe} 1 & 70.06(10) & \mathrm{O} 2-\mathrm{C} 12-\mathrm{H} 12 \mathrm{C} & 109.5 \\ \mathrm{C} 3-\mathrm{C} 2-\mathrm{H} 2 \mathrm{~A} & 126.3 & \mathrm{H} 12 \mathrm{~A}-\mathrm{C} 12-\mathrm{H} 12 \mathrm{C} & 109.5 \\ \mathrm{C} 1-\mathrm{C} 2-\mathrm{H} 2 \mathrm{~A} & 126.3 & \mathrm{H} 12 \mathrm{~B}-\mathrm{C} 12-\mathrm{H} 12 \mathrm{C} & 109.5 \\ \mathrm{Fe} 1-\mathrm{C} 2-\mathrm{H} 2 \mathrm{~A} & 126.3 & \mathrm{O} 3-\mathrm{C} 23-\mathrm{O} 4 & 124.43(17) \\ \mathrm{C} 4-\mathrm{C} 3-\mathrm{C} 2 & 108.49(18) & \mathrm{O} 3-\mathrm{C} 23-\mathrm{N} 2 & 125.99(17) \\ \mathrm{C} 4-\mathrm{C} 3-\mathrm{Fe} 1 & 69.48(12) & \mathrm{O} 4-\mathrm{C} 23-\mathrm{N} 2 & 109.58(15) \\ \mathrm{C} 2-\mathrm{C} 3-\mathrm{Fe} 1 & \mathrm{O} 4-\mathrm{C} 24-\mathrm{H} 24 \mathrm{~A} & 109.5 \\ \mathrm{C} 4-\mathrm{C} 3-\mathrm{H} 3 & 10.15(11) & \mathrm{O} 4-\mathrm{C} 24-\mathrm{H} 24 \mathrm{~B} & 109.5 \\ \mathrm{C} 2-\mathrm{C} 3-\mathrm{H} 3 & 125.8 & \mathrm{H} 24 \mathrm{~A}-\mathrm{C} 24-\mathrm{H} 24 \mathrm{~B} & 109.5 \\ \mathrm{Fe} 1-\mathrm{C} 3-\mathrm{H} 3 & 125.8 & \mathrm{O} 4-\mathrm{C} 24-\mathrm{H} 24 \mathrm{C} & 109.5 \\ \mathrm{C} 3-\mathrm{C} 4-\mathrm{C} 5 & 108.27(18) & \mathrm{H} 24 \mathrm{~A}-\mathrm{C} 24-\mathrm{H} 24 \mathrm{C} & 109.5 \\ \mathrm{C} 3-\mathrm{C} 4-\mathrm{Fe} 1 & 70.04(11) & \mathrm{C} 11-\mathrm{N} 1-\mathrm{C} 1 & 124.97(15) \\ \mathrm{C} 5-\mathrm{C} 4-\mathrm{Fe} 1 & 70.02(11) & \mathrm{C} 11-\mathrm{N} 1-\mathrm{H} 1 & 118.5(14) \\ \mathrm{C} 3-\mathrm{C} 4-\mathrm{H} 4 & 125.9 & \mathrm{C} 1-\mathrm{N} 1-\mathrm{H} 1 & 116.5(14) \\ \mathrm{C} 5-\mathrm{C} 4-\mathrm{H} 4 & 125.9 & \mathrm{C} 23-\mathrm{N} 2-\mathrm{C} 13 & 125.23(15) \\ \mathrm{Fe} 1-\mathrm{C} 4-\mathrm{H} 4 & 125.9 & \mathrm{C} 23-\mathrm{N} 2-\mathrm{H} 2 & 118.8(15) \\ \mathrm{C} 1-\mathrm{C} 5-\mathrm{C} 4 & 107.56(18) & \mathrm{C} 11-\mathrm{O} 2-\mathrm{C} 12 & 115.9(15) \\ \mathrm{C} 1-\mathrm{C} 5-\mathrm{Fe} 1 & 70.37(10) & \mathrm{C} 23-\mathrm{O} 4-\mathrm{C} 24 & 116.42(17) \\ \mathrm{C} 4-\mathrm{C} 5-\mathrm{Fe} 1 & 69.16(11) & & \\ \mathrm{C} 1-\mathrm{C} 5-\mathrm{H} 5 & 126.2 & & \end{array}$

Hydrogen-bond geometry $\left(\AA,{ }^{\circ}\right)$

\begin{tabular}{lllll}
\hline$D-\mathrm{H} \cdots A$ & $D-\mathrm{H}$ & $\mathrm{H} \cdots A$ & $D \cdots A$ & $D-\mathrm{H} \cdots A$ \\
\hline $\mathrm{N} 1-\mathrm{H} 1 \cdots \mathrm{O} 3^{\mathrm{i}}$ & $0.82(2)$ & $2.18(2)$ & $2.971(2)$ & $162.5(19)$ \\
$\mathrm{N} 2-\mathrm{H} 2 \cdots \mathrm{O} 1$ & $0.80(2)$ & $2.18(2)$ & $2.9605(19)$ & $166(2)$ \\
\hline
\end{tabular}

Symmetry code: (i) $x+1, y, z$. 